\title{
A comparison of anisotropic and isotropic grain growth simulation results of Uranium dioxide and Copper
}

\author{
John-Michael H. Bradley
}

August 2015

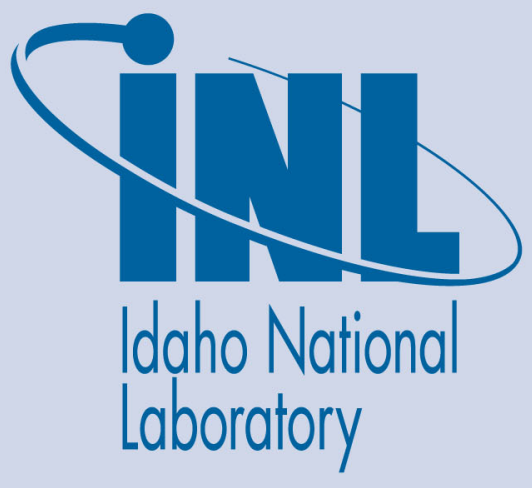

The INL is a U.S. Department of Energy National Laboratory operated by Battelle Energy Alliance 


\title{
A comparison of anisotropic and isotropic grain growth simulation results of Uranium dioxide and Copper
}

\author{
John-Michael H. Bradley
}

\author{
August 2015 \\ Idaho National Laboratory \\ Idaho Falls, Idaho 83415 \\ http://www.inl.gov \\ Prepared for the \\ U.S. Department of Energy \\ Under DOE Idaho Operations Office \\ Contract DE-AC07-05ID14517
}


A comparison of anisotropic and isotropic grain growth simulation results of Uranium dioxide and Copper

\author{
John-Michael H. Bradley', a) \\ Department of Physics, Brigham Young University - Idaho
}

(Dated: 25 August 2015)

a)bra10004@byui.edu 


\begin{abstract}
Grain boundaries migrate to reduce the total grain boundary energy in a material. However, grain boundary energy is anisotropic, such that some grain boundaries are less energy favorable in the system and tend to disappear. Idaho National Laboratory's (INL) mesoscale fuel performance code MARMOT currently assumes isotropic (non-directionally dependent) grain boundary energy in all phase field simulations of grain boundary migration. As a preliminary step to incorporating fully anisotropic grain boundary energies into MARMOT, a comparison of the results of grain growth simulations for $\mathrm{UO}_{2}$ nuclear fuels utilizing the old isotropic MARMOT code and new code incorporating anisotropic grain boundary energies was performed. Comparisons of Copper simulations were also performed. The results analyzed were from simple circle and thumb-shaped grain test cases, and polycrystalline systems using artificially generated initial conditions. The metrics used to compare the grains from isotropic and anisotropic cases include grain area and average grain boundary energy. Anisotropic behavior was observed in the systems of circle and thumb-shaped grains. Polycrystalline systems do not behave as expected, suggesting missing terms in the equation implemented in MARMOT which governs the microstructural evolution of the grain boundaries.
\end{abstract}

\title{
I. INTRODUCTION
}

\section{A. Background}

Different crystalline materials have different properties, but these are often dependent not only on the chemical composition of the material, but also on its microstructure. ${ }^{1}$ Properties of $\mathrm{UO}_{2}$, a common nuclear fuel, which are dependent upon its microstructure include thermal conductivity, mechanical strength, and fission gas release. ${ }^{2}$ Precise calculations of these properties are necessary to create accurate engineering scale models of nuclear fuels. This creates a need for accurate modeling and simulation the fuel's microstructure.

MARMOT is Idaho National Laboratory's mesoscale nuclear fuel performance code. It uses a phase field model to predict the microstructure evolution of polycrystalline materials. These mesoscale models require inputs from the atomic scale (molecular dynamics simulations). The properties MARMOT calculates, such as thermal conductivity and fission gas release, are fed into the engineering scale models. These models may be used to run simulations of entire fuel rods enabling the simulation of entire reactors. 


\section{B. Isotropic vs. anisotropic}

MARMOT employs some approximations by treating certain anisotropic (directionally dependent) parameters as isotropic (non-directionally dependent), including grain boundary energy. The error caused by this approximation is unclear.

Anisotropic grain boundary energy was noted by as early as 1947 by $\mathrm{Smith}^{3}$, and has been demonstrated both in experiment and simulation. ${ }^{4}$ Kasaryan et al., ${ }^{5}$ have shown that anisotropic grain boundary energy, which is a function of grain misorientation and inclination, affects the grain growth kinetics (such as the average grain area vs. time), misorientation distribution, and edge distribution in 2-D polycrystal simulations. The misorientation distribution is related to the ratio of low to high energy grain boundaries, and it was shown by Kazaryan et al. that in a system of anisotropic grain boundary energy this ratio increases with time. Edge distribution refers to the frequency at which grains with a certain number of edges occur. Kazaryan observed that few sided grains, grains with less than 6 sides, occured with much greater frequency in systems of anisotropic grain boundary energy than in isotropic systems, or systems of just anisotropic mobility. Dillon and Rohrer demonstrated using multiple polycrystalline materials that grain boundaries with higher energy are preferrentially eliminated, leading to an anisotropic grain boundary character distribution. ${ }^{4}$ These observations and those by Kazaryan suggest that treating grain boundary energy as anisotropic is important for realistic simulations. Correctly modeling these anisotropic behaviors will enable more accurate modeling of nuclear fuels at the engineering scale.

\section{A function for grain boundary energy}

Upon consideration of the importance of grain boundary energy anisotropy, and in light of the recent discovery of a close scaling between the energies of 388 grain boundaries of four face-centered cubic metals ${ }^{6,7}$, Bulatov wrote a fitting function of five degrees of freedom for FCC metals to calculate the grain boundary energy as a function of the misorientation and inclination of adjacent grains. ${ }^{8}$ Tolliver recently implemented the code in MARMOT and is currently optimizing it for use. ${ }^{9}$ This code requires validation. 


\section{CODE VALIDATION}

All software that models and simulates real phenomena needs to be validated. This means that it must be shown to correctly represent physical behavior. There are several ways this can be done. One of the more robust ways is to compare simulation results against experimental data. Additionally, If an analytical solution is available which has already been validated, one may compare against that. In our problem there is no readily available analytical solution. However, if we wish to perform partial validation, we may do so qualitatively, before comparing against experimental data. Simulations may be performed to observe for the predicted anisotropic behavior and these results may be compared against isotropic simulations. If these predicted behaviors are observed, one may confidently move on from these preliminary tests to more in-depth methods of validation. This is the approach which has been taken here.

\section{A. Preliminary tests}

Preliminary to a full comparison against experimental data, multiple simulations were performed to test that the model produced anisotropic behavior. There are many behaviors which are evidence of grain boundary energy anisotropy. One common observation is that grain shape in single and double grain systems should evolve with time. This is because differing inclinations around the grain boundary perimeter cause different energies around the grain boundary. A second behavior is that grains with different average grain boundary energy should shrink at different rates (this difference in energy is caused by different misorientations and/or inclinations). These two behaviors are the result of the relation between the velocity of the grain boundary and the grain boundary energy, ${ }^{10}$

$$
v_{g b, n}=-\mu_{g b}(\phi)\left\{\frac{1}{R_{1}}\left[\sigma_{g b}(\phi)+\frac{\partial^{2} \sigma_{g b}(\phi)}{\partial \alpha_{1}^{2}}\right]+\frac{1}{R_{2}}\left[\sigma_{g b}(\phi)+\frac{\partial^{2} \sigma_{g b}(\phi)}{\partial \alpha_{2}^{2}}\right]\right\}
$$

where $\mu_{g b}$ is the grain boundary mobility, $1 / R$ is the mean curvature (of the grain boundary), $\alpha$ is the angle between the grain boundary normal and a vector of length $R$ stretching from the grain center to the grain boundary, and $\sigma_{g b}$ is the grain boundary energy.

A third behavior observed in systems of anisotropic grain boundary energy is that the average grain boundary energy in polycrystalline systems should decrease with time. As the grain microstructure evolves, the high energy boundaries shrink preferentially. ${ }^{4}$ This is 
fundamentally due to the tendency of all physical systems to seek the lowest possible energy state.

\section{Circle-shaped grains}

In order to observe for changing grain shape, two simulations of circle grains, one isotropic and one anisotropic, were performed. The goal of the comparison was for the circle-shaped grain in the anisotropic system to develop eccentricity with time due to differening inclinations about the grain boundary perimiter. The grain had a misorientation defined by a 30 degree rotation about the x-axis. The tests were run for a simulated 2.485 years at a temperature of 1000 K. See Figs. 1 and 2.

\section{Thumb-shaped grains}

In order to observe differing rates of collapse between the two grains due to the differing inclinations (and therefore differing energies), one simulation of thumb-shaped grains was performed. It consisted of two thumb-shaped grains, one situated horizontally and the other vertically, each with a misorientation defined by a 30 degree rotation about the $\mathrm{x}$-axis. The simulated temperature was $1000 \mathrm{~K}$, and the simulations were run until the grains collapsed completely. See Figs. 4 and 5.

\section{Polycrystalline systems}

To test for decreasing average grain boundary energy, several simulations were performed of systems of 100 and 1000 grains. Each simulation was run once with isotropic grain boundary energy, and once with anisotropic grain boundary energy. The simulations of 100 grains were performed of both Copper and Uranium dioxide, and the 1000 grain simulation of Copper. (The simulations of $\mathrm{Cu}$ served as a control to ensure that any unexpected behavior

observed in the simulations of $\mathrm{UO}_{2}$ was not due to faulty material specific parameters used in Bulatov's function.)

Throughout each simulation the average grain boundary energy was calculated by dividing the total grain boundary energy by the grain boundary area. The grain boundary energy 
was calculated in all systems using the new code implemented by Tolliver. ${ }^{9}$ This was also done in the isotropic systems, but in these simulations only the constant isotropic energies were used to affect the evolution of the microstructure. Thus, the average grain boundary energy of both systems (isotropic and anisotropic), as calculated by the new code, could be compared to show if the ratio of low energy boundaries to high energy boundaries decreased with time, or equivalently, if the average grain boundary energy decreased with time.

\section{RESULTS AND DISCUSSION}

\section{A. Circle-shaped grains}

The results of the circle-shaped grain simulations may be seen in Figs. 1 and 2. The eccentricity of the grain in the anisotropic case suggests correctly simulated anisotropic behavior. The anisotropic case has a higher average grain boundary energy which is illustrated by its more rapid collapse. The greater rate of collapse is explained by Eqn. 1-whichever grain has a higher average grain boundary energy will grow or collapse at a greater rate.

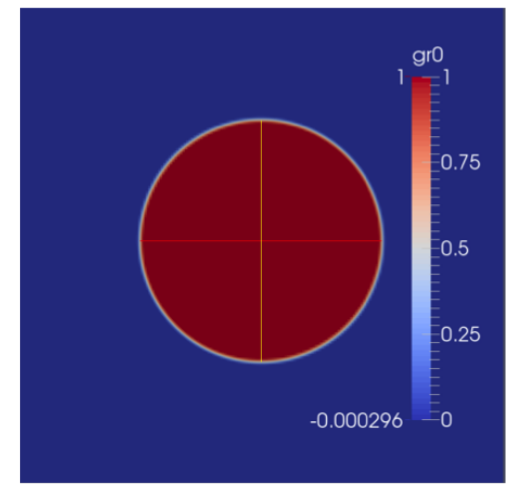

(a) Isotropic

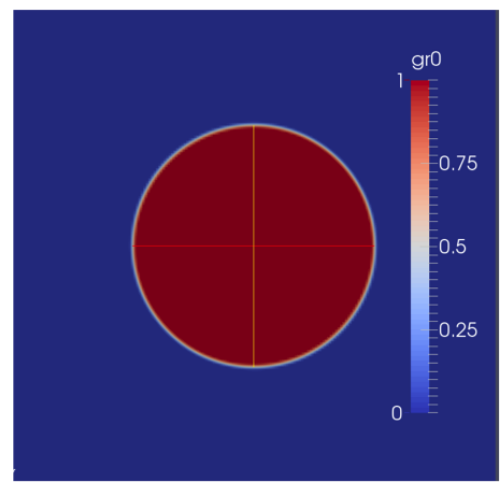

(b) Anisotropic

FIG. 1: $\mathrm{UO}_{2}$ circle-grain initial conditions, time $=0 \mathrm{yr}$, temperature $=1000 \mathrm{~K}$. Bar scale is measuring presence or absence of the circle-shaped grain variable gr0.

\section{B. Thumb-shaped grains}

It was hypothesized that one thumb-shaped grain would shrink faster than the other, because of their different inclinations at the grain tips. (Inclination can be measured in 


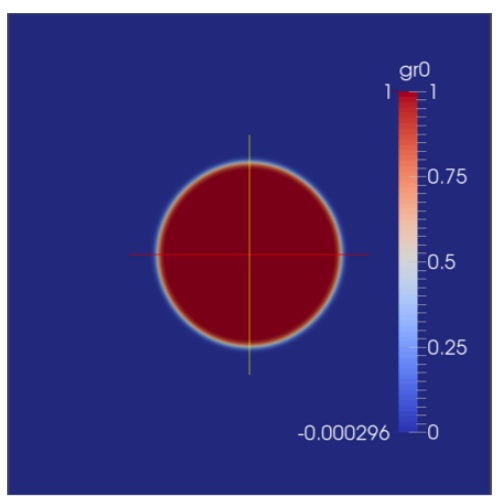

(a) Isotropic

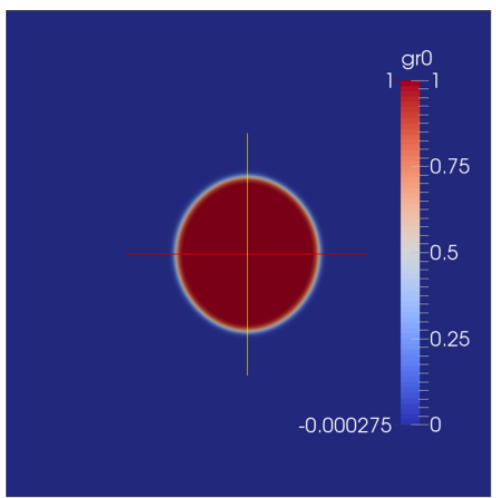

(b) Anisotropic

FIG. 2: $\mathrm{UO}_{2}$ circle-grains at time $=2.485 \mathrm{yr}$. The grain with anisotropic grain boundary energy has developed slight eccentricity as expected. Bar scale is measuring presence or absence of the circle-shaped grain variable gr0.

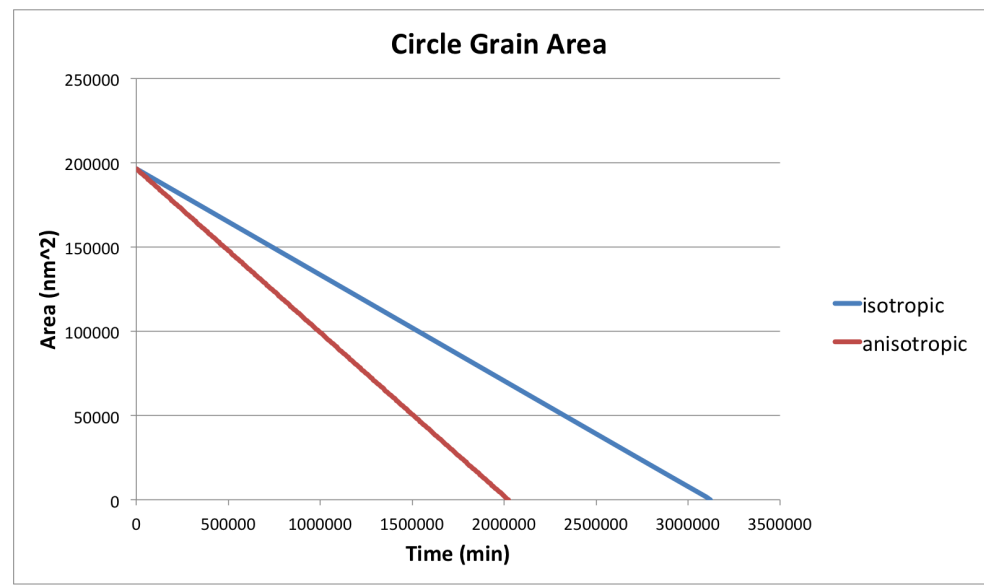

FIG. 3: Area of isotropic and anisotropic $\mathrm{UO}_{2}$ circle-shaped grains with respect to time. Simulation parameters: temperature $=1000 \mathrm{~K}$, time $=0-2.485 \mathrm{yr}$.

different ways, but is related to the angle of the grain boundary with respect to the lattices of surrounding grains.) However, as can be seen in Fig. 5, it was observed that the grains shrunk at approximately the same rate. This suggests that, even though the energies at the tips of the grains are different, the average grain boundary energy of the parts of each thumbshaped grain with non-zero curvature are approximately equivalent. (This is a realistic assumption given that the side of the grains with zero curvature should not contribute to the rate of grain collapse.) Additional analysis indeed showed that the semi-circle portions of the thumb-shaped grains had the same average grain boundary energy (See Fig. 6). 


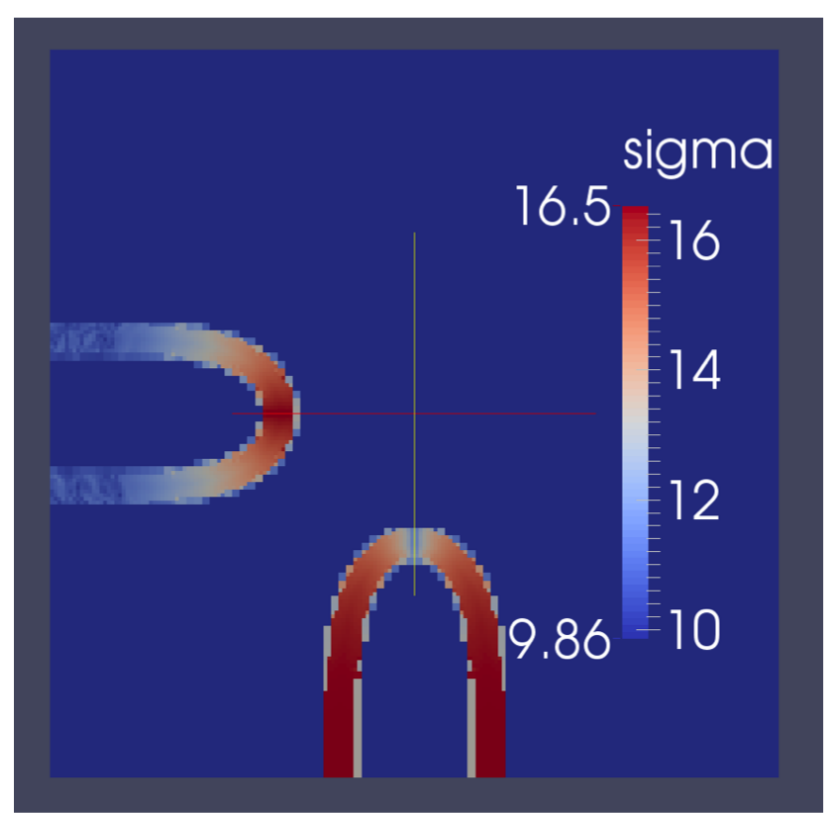

FIG. 4: Grain boundary energy of thumb-shaped grains. Here sigma represents the grain boundary energy in electron volts per square nanometer. Both grains have the same misorientation (30 degrees about the x-axis). The different energies are the result of different inclinations.

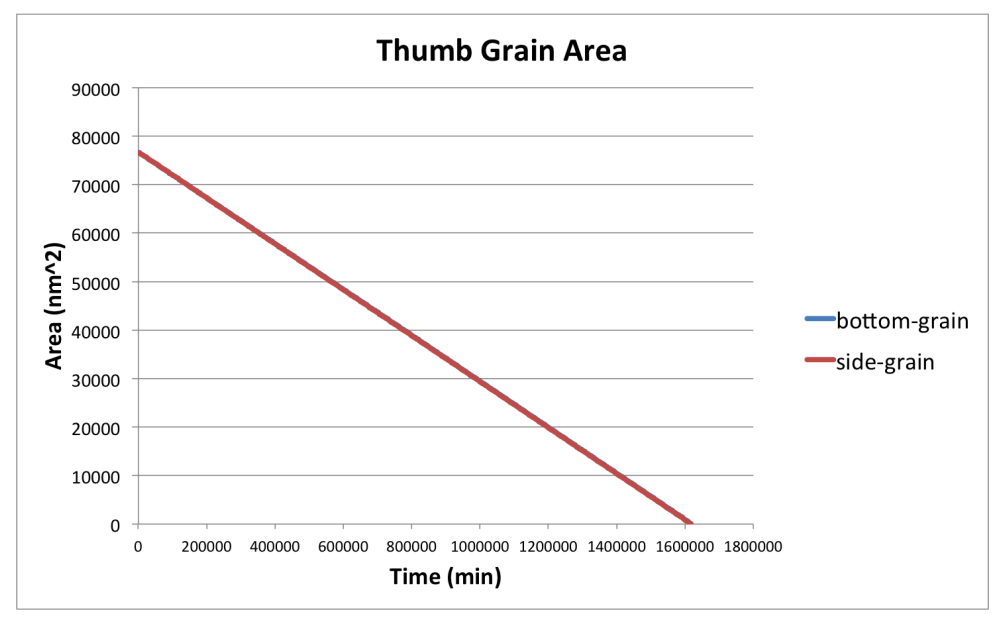

FIG. 5: Area of thumb-shaped grains vs. time. Both grains unexpectedly collapse as the same rate, the data points for grain 2 lying directly above the data points of grain 1 .

This increases the possiblity that the phenomena of both grains shrinking at the same rate is due purely to their semi-circlular ends, not an absence of inclination-dependent anisotropy. 


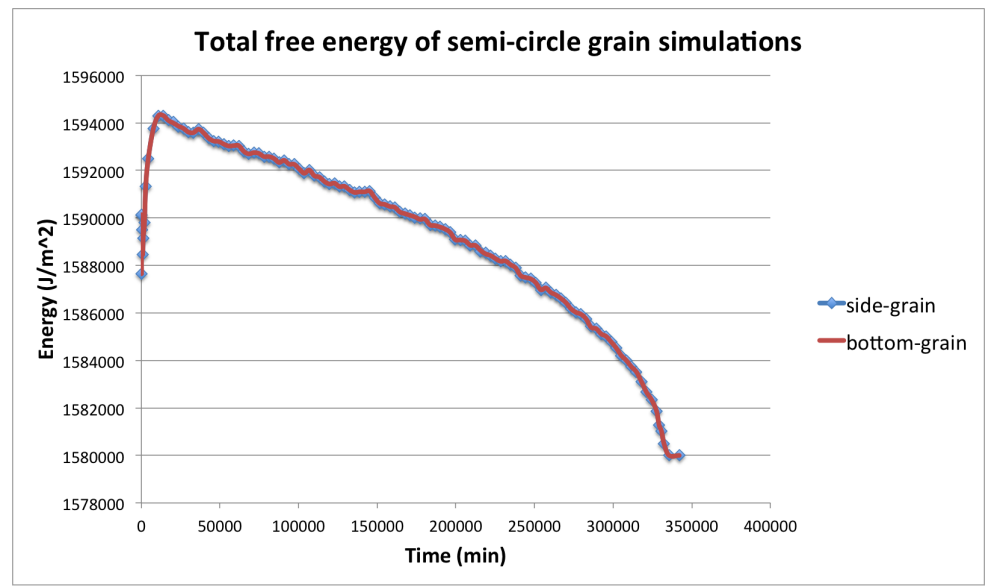

FIG. 6: The total free energy of the semi-circular portions of the thumb-shaped grains vs. time. This simulation shows that the tips of the thumb-shaped grains have the same average grain boundary energy.

\section{Polycrystalline systems}

We expected to see the average grain boundary energy in the anisotropic systems decrease with time, and that in the isotropic systems it would vary slightly around the initial average grain boundary energy. Instead, the average grain boundary energy displayed rather unpredictable trends (See Figs. 7-9). We believe that this may be due to incomplete

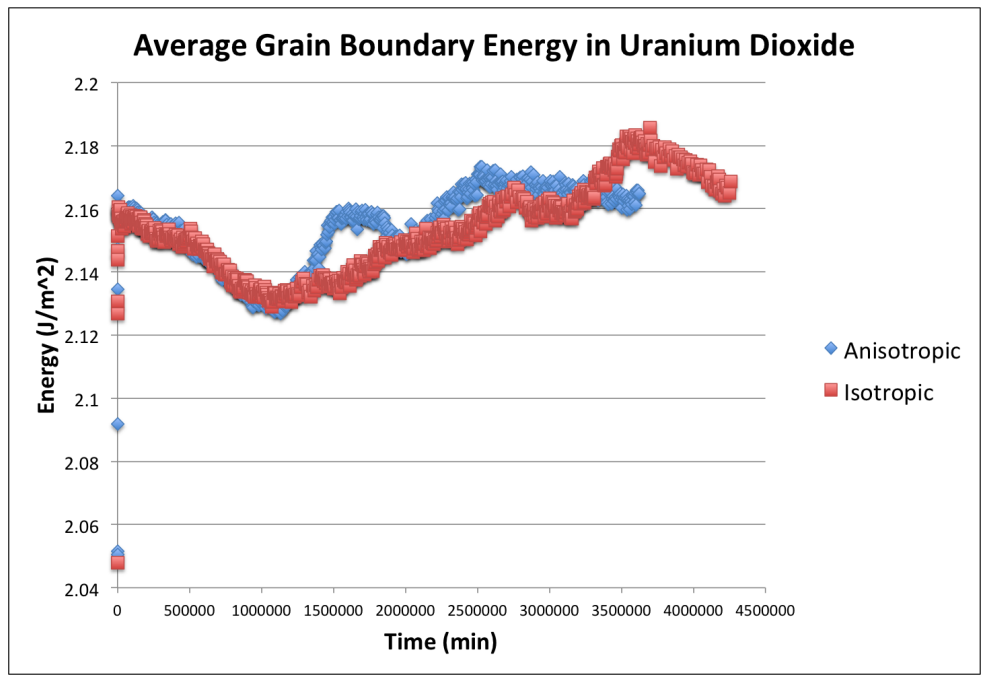

FIG. 7: Average grain boundary energy in Uranium dioxide. 100 grains. Temperature = $1000 \mathrm{~K}$. 


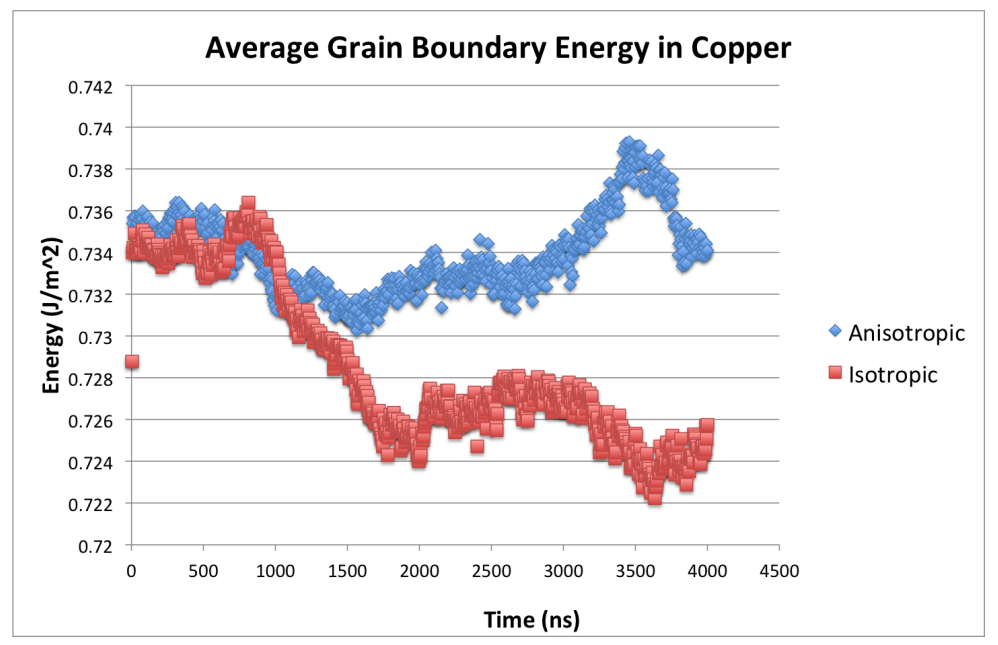

FIG. 8: Average grain boundary energy in Copper. 100 grains. Temperature $=500 \mathrm{~K}$.

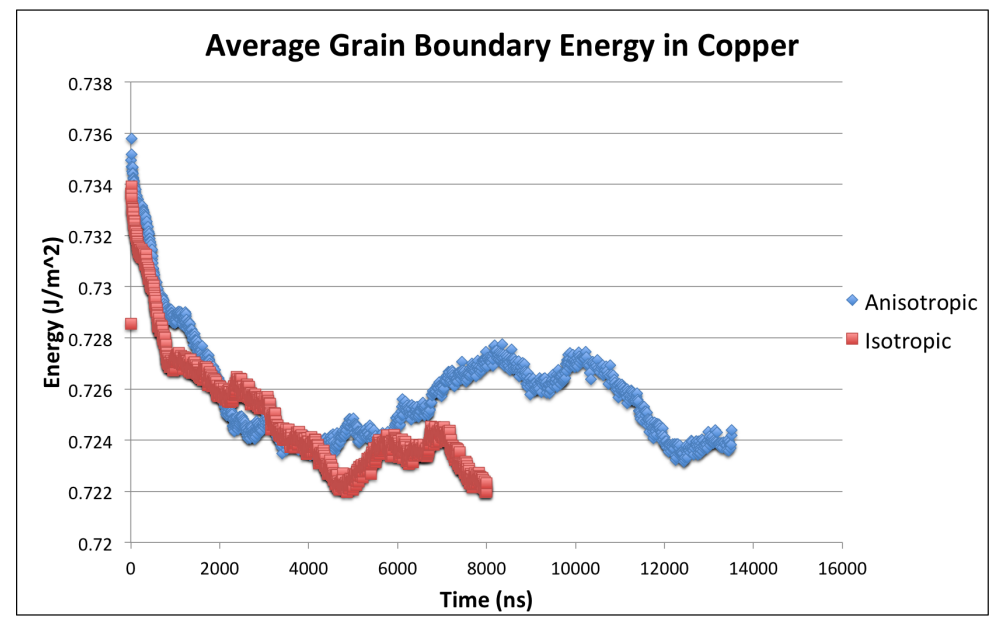

FIG. 9: Average grain boundary energy in Copper. 1000 grains. Temperature $=500 \mathrm{~K}$.

MARMOT Kernels. A Kernel is a piece of code that represents one term in the partial differential equation used to represent the system. Because MARMOT has historically only treated grain boundary energy as isotropic, the terms representing the anisotropic grain boundary energy in the Ginzburg-Landau equation (see Ref. 10), the phase field equation governing the microstructural evolution of the system, were not included.

\section{CONCLUSIONS AND FUTURE WORK}

Grains collapse and change shape as expected in simulations of one and two grains. Average grain boundary energy in larger systems does not evolve as expected, possibly due 
to incomplete Kernels. In general, the simulations performed are not sufficient for validation, and more investigations are required. New Kernels need to be added to MARMOT, and similar simulations run to see if the average grain boundary energy then behaves as expected. An investigation as to why the average grain boundary in the isotropic case decreases with time is also needed (it should not be decreasing). These actions need to be taken before a comparison is made against experimental data.

Once the above mentioned investigations are made, a comparison of the grain boundary character distributions and the grain boundary energy distributions of anisotropic grain boundary simulations and X-ray diffraction data from Los Alamos National Laboratory will be performed. Uranium dioxide pre, in-situ, and post grain growth X-ray diffraction microscopy data will be compared with anisotropic grain boundary energy simulations begun from real initial conditions. This will permit a robust validation of the new MARMOT code.

\section{ACKNOWLEDGMENTS}

Funding for this project was provided by the Department of Energy Office of Science and the Department of Energy NEAMS program.

\section{REFERENCES}

${ }^{1}$ G. Gottstein and L. Shvindkermank, Grain boundary migration in metals: thermodynamics, kinetics, applications (CRC Press, Boca Raton, FL, 1999).

${ }^{2} \mathrm{~T}$. Harbison, Anisotropic grain boundary energy function for uranium dioxide, BYU-Idaho (2015).

${ }^{3}$ C. Smith, "Introduction to grains, phases, and interfaces-an interpretation of microstructure," Trans. AIME 175, 15-51 (1948).

${ }^{4}$ S. J. Dillon and G. S. Rohrer, "Mechanism for the development of anisotropic grain boundary character distributions during normal grain growth," Acta Mater. 57, 1 - 7 (2009).

${ }^{5}$ A. Kazaryan, Y. Wang, S. Dregia, and B. Patton, "Grain growth in anisotropic systems: comparison of effects of energy and mobility," Acta Mater. 50, 2491 - 2502 (2002).

${ }^{6}$ D. L. Olmsted, S. M. Foiles, and E. A. Holm, "Survey of computed grain boundary 
properties in face-centered cubic metals: I. grain boundary energy," Acta Mater. 57, 3694 - 3703 (2009).

${ }^{7}$ E. A. Holm, D. L. Olmsted, and S. M. Foiles, "Comparing grain boundary energies in face-centered cubic metals: Al, au, cu and ni," Scr. Mater. 63, 905 - 908 (2010).

${ }^{8} \mathrm{~V}$. Bulatov, B. Reed, and M. Kumar, "Grain boundary energy function for fcc metals," Acta Mater. 65, 161 - 175 (2014).

${ }^{9} \mathrm{~J}$. Tolliver, "Incorporating anisotropic grain boundary energy into a grain growth model," Unpublished.

${ }^{10}$ N. Moelans, B. Blanpain, and P. Wollants, "Quantitative analysis of grain boundary properties in a generalized phase field model for grain growth in anisotropic systems," Phys. Rev. B 78, 024113 (2008). 